$\mathrm{IC} / 91 / 384$

November, 1991

\title{
Modified Black Holes in Two Dimensional Gravity
}

\author{
Noureddine Mohammedi \\ International Centre for Theoretical Physics \\ P. O. Box 586, 34100 Trieste, Italy.
}

\begin{abstract}
The $S L(2, R) / U(1)$ gauged WZWN model is modified by a topological term and the accompanying change in the geometry of the two dimensional target space is determined. The possibility of this additional term arises from a symmetry in the general formalism of gauging an isometry subgroup of a non-linear sigma model with an antisymmetric tensor. It is shown, in particular, that the space-time exhibits some general singularities for which the recently found black hole is just a special case. From a conformal field theory point of view and for special values of the unitary representations of $S L(2, R)$, this topological term can be interpreted as a small perturbation by a $(1,1)$ conformal operator of the gauged WZWN action.
\end{abstract}

\footnotetext{
${ }^{1}$ nour@itsictp.bitnet
} 


\section{Introduction}

Conformal field theories with central charge $c=1$, when coupled to twodimensional gravity provide interesting toy models for the study of string theories. Perhaps the most appealing feature of these $c=1$ theories is their ability to describe strings in non-critical dimensions in a non-perturbative way [1-18]. This is due to the recent success of the matrix model approach to non-critical strings. Another property of these theories is their target space-time interpretation as critical string theories propagating in non-trivial $1+1$ dimensional backgrounds [6-9]. In particular, the metric of this two-dimensional target space exhibits a black hole singularity just as the Schwarzschild black hole in four dimensions $[19,20]$. Of great importance, however, is the possibility of viewing these singularities as arising from a modular invariant $S L(2, R) / U(1)$ coset WZWN model [19].

In section two of this paper, we explore the change in the geometry of the two dimensional target space due a modification by a topological term of the gauged WZWN action. The possibility of this additional term stems from the general formalism of gauging an arbitrary isometry subgoup of a nonlinear sigma model with an antisymmetric tensor. We find that, in general, the scalar curvature is singular whenever the quantity

$\frac{1}{16(1-u v)^{2}}\left[4+2(u v-4) \partial_{u} X \partial_{v} X+4 u \partial_{u} X-4 v \partial_{v} X+u^{2}\left(\partial_{u} X\right)^{2}+v^{2}\left(\partial_{v} X\right)^{2}\right]$

has zeros or is undefined. Here $(u, v)$ are the target space coordinates and $X(u, v)$ is a scalar function introduced by the topological term.

We also consider, in section three, the effects of the additional term on the conformal field theory of the black hole as described by the $S L(2, R) / U(1)$ coset theory. We show that when the unitary representations $\mid l, m>$ of the universal cover $\widetilde{S L}(2, R)$ and the level $k$ satisfy

$$
-\frac{l(l+1)}{k-2}+\frac{m^{2}}{4 k}=0,
$$

the extra topological term is a conformal operator of dimension $(1,1)$. Therefore it can be considered as a kind of perturbation of the ordinary $S L(2, R) / U(1)$ coset model. 


\section{The Topological Term}

The non-linear sigma model with a Wess-Zumino term as given by

$$
S=\frac{1}{2 \pi} \int \mathrm{d}^{2} x\left(\sqrt{\gamma} \gamma^{\mu \nu} G_{i j}+\epsilon^{\mu \nu} B_{i j}\right) \partial_{\mu} \phi^{i} \partial_{\nu} \phi^{j}
$$

is invariant under the global $U(1)$ isometry transformations, ( $\alpha$ is constant),

$$
\delta \phi^{i}=\alpha K^{i}(\phi)
$$

provided that $K^{i}$ is a Killing vector of the metric $G_{i j},\left(\nabla_{(i} K_{j)}=0\right)$, and

$$
\partial_{l} H_{i j k} K^{l}+H_{l j k} \partial_{i} K^{l}+H_{i l k} \partial_{j} K^{l}+H_{i j l} \partial_{k} K^{l}=0
$$

The torsion is defined by $H_{i j k}=\frac{3}{2} \partial_{[i} B_{j k]}$. It follows that the antisymmetric tensor $B_{i j}$ satisfies

$$
\partial_{l} B_{i j} K^{l}+B_{l j} \partial_{i} K^{l}+B_{i l} \partial_{j} K^{l}=\nabla_{i} L_{j}-\nabla_{j} L_{i}
$$

for some vector $L_{i}[21]$.

The above transformation can be made local, $(\alpha=\alpha(x))$, b y the introduction of a $U(1)$ gauge field $A_{\mu}$. Then the gauged action takes the form $[22,23]$

$$
S_{g}=\frac{1}{2 \pi} \int d^{2} x\left\{\sqrt{\gamma} \gamma^{\mu \nu} G_{i j} D_{\mu} \phi^{i} D_{\nu} \phi^{j}+\epsilon^{\mu \nu} B_{i j} \partial_{\mu} \phi^{i} \partial_{\nu} \phi^{j}-2 \epsilon^{\mu \nu} C_{i} A_{\mu} \partial_{\nu} \phi^{i}\right\}
$$

where

$$
\begin{aligned}
D_{\mu} \phi^{i} & =\partial_{\mu} \phi^{i}+A_{\mu} K^{i} \\
C_{i} & =B_{i j} K^{j}+L_{i} .
\end{aligned}
$$

The gauge field $A_{\mu}$ transforms as $\delta A_{\mu}=-\partial_{\mu} \alpha$. The action (2.5) is then invariant under local gauge transformations if $[22,23]$

$$
\begin{gathered}
\partial_{j} C_{i} K^{j}+C_{j} \partial_{i} K^{j}=0 \\
L_{i} K^{i}=0
\end{gathered}
$$

The gauge field apprears only quadratically and with no derivatives. Hence, it can be eliminated via its equations of motion. The resulting theory 
is again a non-linear sigma model with a new metric $\widehat{G}_{i j}$ and an antisymmetric tensor $\widehat{B}_{i j}$ given by

$$
\begin{aligned}
\widehat{G}_{i j} & =G_{i j}-\frac{1}{M}\left(G_{i k} G_{j l} K^{k} K^{l}+C_{i} C_{j}\right) \\
\widehat{B}_{i j} & =B_{i j}+\frac{1}{M}\left(G_{i k} C_{j} K^{k}-G_{j k} C_{i} K^{k}\right),
\end{aligned}
$$

where

$$
M=G_{i j} K^{i} K^{j}
$$

Notice that the new metric $\widehat{G}_{i j}$ would exhibit an explicit singular ity if $M$ has zeros. This is so if the old metric $G_{i j}$ is not positive definite as it is in the case when the non-linear sigma model is defined on a non-compact group manifold. Using Eq.(2.8) we find

$$
\widehat{G}_{i j} K^{j}=0
$$

Therefore due to these null eigenvectors, the metric $\widehat{G}_{i j}$ cannot be inverted and we cannot analyse the singularities of the gauged non-linear sigma model. To overcome this difficulty, a gauge fixing term in the action (2.5) is necessary.

The main remark in this note is that the defining equation for $L_{i}$, in (2.4), is invariant under the shift [22]

$$
L_{i} \rightarrow L_{i}+\partial_{i} \lambda
$$

To guarantee that the gauged action (2.5) is invariant under the above shift, we should modify our action in the following manner

$$
S_{g} \rightarrow S_{g}+\frac{1}{2 \pi} \int \mathrm{d}^{2} x \epsilon^{\mu \nu} F_{\mu \nu} X(\phi)
$$

where $F_{\mu \nu}=\partial_{\mu} A_{\nu}-\partial_{\nu} A_{\mu}$ is the $U(1)$ field strength. The additional term is gauge invariant if

$$
\partial_{i} X K^{i}=0
$$

The gauged action will be invariant under the shift (2.11) provided that $X(\phi)$ undergoes the transformation

$$
X \rightarrow X+\partial_{i} \lambda
$$

Furthermore, the term in $F_{\mu \nu}$ is topological in nature. 
The possibility of the additional term in Eq.(2.12) may also be seen from a different point of view: In the normal coordinate expansion of the gauged action, when the gauge field is taken as a fixed background, terms proportional to $F_{\mu \nu}$ are generated [22]. Indeed, in order to expand the action $S_{g}$ around some classical field $\phi$ in a covariant fashion [24], we introduce the quantum field $\xi^{i}(x)$ which is the tangent at $\phi^{i}$ to the geodesic joining $\phi^{i}$ to $\phi^{i}+\pi^{i}$, where $\pi^{i}$ is a small perturbation around $\phi^{i}$. We find that the first term in the expansion of the $\epsilon^{\mu \nu}$ term in $S_{g}$ is given by [22]

$$
\frac{1}{2 \pi} \int d^{2} x \epsilon^{\mu \nu}\left(H_{i j k} D_{\mu} \phi^{i} D_{\nu} \phi^{j}-C_{k} F_{\mu \nu}\right) \xi^{k} .
$$

It is clear, therefore, that divergences proportional to $F_{\mu \nu}$ will appear upon quantisation. Hence, for a renormalisable theory we should add a term in $F_{\mu \nu}$ to the classical action.

To see the consequences of this additional term on the black hole physics, let us apply our formalism to the $S L(2, R)$ case. The group manifold is parametrised by 2

$$
g=\left(\begin{array}{cc}
a & u \\
-v & \frac{1}{a}(1-u v)
\end{array}\right),
$$

and the $U(1)$ isometry group is generated by

$$
\delta a=2 \alpha a, \delta u=\delta v=0
$$

The gauged WZWN action is given by an expression of the form (2.5), (with the factor $\frac{1}{2 \pi}$ in the front replaced by $-\frac{k}{4 \pi}$ ), and where

$$
\begin{aligned}
G_{i j} & =\left(\begin{array}{ccc}
-\frac{1}{a^{2}}(1-u v) & -\frac{1}{2} \frac{v}{a} & -\frac{1}{2} \frac{u}{a} \\
-\frac{1}{2} \frac{v}{a} & 0 & \frac{1}{2} \\
-\frac{1}{2} \frac{u}{a} & \frac{1}{2} & 0
\end{array}\right) \\
B_{i j} & =\left(\begin{array}{ccc}
0 & 0 & 0 \\
0 & 0 & -\ln a \\
0 & \ln a & 0
\end{array}\right) .
\end{aligned}
$$

Here $a=\phi^{1}, u=\phi^{2}, v=\phi^{3}$, and $L_{i}$ and $C_{i}$ are given by

$$
\begin{aligned}
& L_{1}=C_{1}=0 \\
& L_{2}=C_{2}=2 v \\
& L_{3}=C_{3}=-2 u .
\end{aligned}
$$

\footnotetext{
${ }^{2}$ In this section we are using the conventions of ref.[19].

${ }^{3}$ Throughout this note we will restrict our analyses to gauging the non-compact $U(1)$ subgroup.
} 
Adding the term proportional to $F_{\mu \nu}$, as given in (2.12) with $X$ scaled by a factor $k$, and integrating out the gauge field leads to a non-linear sigma model with a metric $\widehat{G}_{i j}$

$$
\begin{aligned}
\widehat{G}_{u u} & =\frac{1}{2(1-u v)}\left[-v \partial_{u} X-\left(\partial_{u} X\right)^{2}\right] \\
\widehat{G}_{v v} & =\frac{1}{2(1-u v)}\left[u \partial_{v} X-\left(\partial_{v} X\right)^{2}\right] \\
\widehat{G}_{u v} & =\frac{1}{2(1-u v)}\left[1+\frac{1}{2} u \partial_{u} X-\frac{1}{2} v \partial_{v} X-\partial_{u} X \partial_{v} X\right] .
\end{aligned}
$$

The coordinate $a$ has been eliminated via the gauge choice $a^{2}=1-u v[19]$. Notice that by setting $X=0$ one gets the usual black hole metric $[19,20]$.

To illustrate the change in the geometry due to the $F_{\mu \nu}$ term, let us compute the scalar curvature for a particular case for the function $X(u, v)$. First of all we find, using (2.13) and (2.17), that $X$ is a function of the coordinate $u$ and $v$ only. Second of all, the only further restiction that one can have on $X$ is the requirement that it satisfies the equation of a scalar field on the target space, namely

$$
\begin{gathered}
\nabla^{2} X(u, v)=0 \\
=\left(-3 u \partial_{u}-3 v \partial_{v}+2(2-u v) \partial_{u} \partial_{v}-u^{2} \partial_{u}^{2}-v^{2} \partial_{v}^{2}\right) X
\end{gathered}
$$

where the covariant derivative $\nabla$ corresponds to the metric (2.18). The last equation is also sufficient for the vanishing of the one-loop counterterm proportional to $F_{\mu \nu}$ when the the gauge field is considered as a fixed background. This counterterm is calculated from the expansion in $\xi^{i}(x)$ given by

$$
\frac{1}{4 \pi} \int d^{2} x \epsilon^{\mu \nu} F_{\mu \nu}\left(\nabla_{i} \nabla_{j}-\nabla_{i} C_{j}\right) \xi^{i} \xi^{j}
$$

The equation in (2.21) has two particular solutions

$$
\begin{aligned}
& X_{1}(u, v)=x u^{-2}+y v^{-2} \\
& X_{2}(u, v)=z \ln \left(\frac{u}{v}\right)
\end{aligned}
$$

with $x, y$ and $z$ being some constants of integration. As an example, the scalar curvature corresponding to the second solution is given by

$$
R_{2}=\frac{-4\left[u^{2} v^{2}\left(1+4 z+6 z^{2}+4 z^{3}\right)+z^{2}(u v+1)\left(1+2 z+2 z^{2}\right)\right]}{(1-u v)\left(u v+2 z u v+2 z^{2}\right)^{2}} .
$$


In addition to the usual singularity at $u v=1$, there appear another singularity at $\left(u v+2 z u v+2 z^{2}\right)=0$. In general the extra singularities are determined by the curves in $u$ and $v$ for which the determinant of the metric $\widehat{G}_{i j}$ is zero or undefined, as given in (1.1).

\section{The Conformal Field Theory Of The Topological Term}

In what follows we will investigate the effects of the additional term involving $F_{\mu \nu}$ on the conformal field theory of the black hole solution. For this purpose the $S L(2, R)$ WZWN model is parametrised by f

$$
g=e^{\frac{i}{2} \theta_{L} \sigma_{2}} e^{\frac{1}{2} r \sigma_{1}} e^{\frac{i}{2} \theta_{L} \sigma_{3}}
$$

where $\left(r, \theta_{L}, \theta_{R}\right)$ are real Euler coordinates and $\sigma_{i}$ are the Pauli matrices. The local gauge transformations correspond to

$$
\delta \theta_{L}=\delta \theta_{R}=\alpha, \delta r=0
$$

The gauged $S L(2, R)$ action takes the form

$$
\begin{aligned}
S=S_{w z w n}\left[r, \theta_{L}, \theta_{R}\right] & +\frac{k}{2 \pi} \int d^{2} z\left[A\left(\bar{\partial} \theta_{R}+\cosh r \bar{\partial} \theta_{L}\right)\right. \\
& \left.+\bar{A}\left(\partial \theta_{L}+\cosh r \partial \theta_{R}\right)-\bar{A} A(\cosh r+1)\right]
\end{aligned}
$$

where the action $S_{w z w n}$ is given by

$$
S_{w z w n}=\frac{k}{4 \pi} \int d^{2} z\left(\bar{\partial} r \partial r-\bar{\partial} \theta_{L} \partial \theta_{L}-\bar{\partial} \theta_{R} \partial \theta_{R}-2 \cosh r \bar{\partial} \theta_{L} \partial \theta_{R}\right)
$$

The gauge field $A=(A, \bar{A})$ is traded for two complex scalars $\phi_{L}$ and $\phi_{R}$ $\left(\phi_{L}=\phi_{R}^{*}\right)$ through [25]

$$
A=\partial \phi_{L}, \quad \bar{A}=\bar{\partial} \theta_{R} .
$$

By redefining the fields as [26]

$$
\theta_{L} \rightarrow \theta_{L}+\phi_{L}, \quad, \quad \theta_{R} \rightarrow \theta_{R}+\phi_{R}
$$

\footnotetext{
${ }^{4}$ In this section we will follow the notation of ref.[26].
} 
one finds that the gauge fixed action has a dependence on $\phi_{L}$ and $\phi_{R}$ through their difference $\phi=\phi_{L}-\phi_{R}$ only, and is given by

$$
S_{g f}=S_{w z w n}\left[r, \theta_{L}, \theta_{R}\right]+S[\phi]+S[b, c],
$$

where $S[\phi]$ describes a free scalar field

$$
S[\phi]=-\frac{k}{4 \pi} \int d^{2} z \partial \phi \bar{\partial} \phi
$$

The Jacobian of the gauge fixing is given by a $(1,0)$ ghost system represented by the action

$$
S[b, c]=\int d^{2} z(b \bar{\partial} c+\bar{b} \partial \bar{c})
$$

The energy momentum tensor corresponding to this action is given by

$$
T(z)=\frac{1}{k-2} \eta_{a b} J^{a} J^{b}+\frac{k}{4} \partial \phi \partial \phi+b \partial c,
$$

and the $S L(2, R)$ currents are given by

$$
\begin{aligned}
J^{3}(z) & =k\left(\partial \theta_{L}+\cosh r \partial \theta_{R}\right) \\
J^{ \pm}(z) & =k e^{ \pm i \theta_{L}}\left(\partial r \pm i \sinh r \partial \theta_{R}\right) .
\end{aligned}
$$

The Virasoro algebra generated by $T(z)$ has a central charge

$$
c=\frac{3 k}{k-2}-1
$$

The primary fields of this Euclidean coset theory have the form [26]

$$
T_{m n}^{l}\left(r, \theta_{L}, \theta_{R}\right)=P_{\omega_{L} \omega_{R}}^{l}(\cosh r) e^{i \omega_{L} \theta_{L}+i \omega_{R} \theta_{R}}
$$

where the quantum numbers $\omega_{L}$ and $\omega_{R}$ are the eigenvalues of $J_{0}^{3}$ and $\bar{J}_{0}^{3}$, respectively and $l$ labels the $S L(2, R)$ isospin. The functions $P_{\omega_{L} \omega_{R}}^{l}$ are the Jacobi functions, and $\omega_{L}$ and $\omega_{R}$ take their values on the $m \times n$ lattice

$$
\omega_{L}=\frac{1}{2}(m+n k) \quad, \quad \omega_{R}=-\frac{1}{2}(m-n k) .
$$

The integers $m$ and $n$ are interpreted, respectively, as the discrete momentum and the winding number of the string in the $\theta=\frac{1}{2}\left(\theta_{L}-\theta_{R}\right)$ direction [26]. 
The vertex operators $T_{m n}^{l}$ are eigenfunctions of the Virasoro operators $L_{0}$ and $\bar{L}_{0}$, which are represented by the differential operators

$$
\begin{aligned}
& L_{0}=-\frac{\triangle_{0}}{k-2}-\frac{1}{k} \frac{\partial}{\partial \theta_{L}^{2}} \\
& \bar{L}_{0}=-\frac{\triangle_{0}}{k-2}-\frac{1}{k} \frac{\partial}{\partial \theta_{R}^{2}},
\end{aligned}
$$

with $\triangle_{0}$ being the Casimir (or the Laplacian) on the group manifold and is given by

$$
\triangle_{0}=\frac{\partial^{2}}{\partial r^{2}}+\cosh r \frac{\partial}{\partial r}+\frac{1}{\sinh ^{2} r}\left(\frac{\partial^{2}}{\partial \theta_{L}^{2}}-2 \cosh r \frac{\partial^{2}}{\partial \theta_{L} \partial \theta_{R}}+\frac{\partial^{2}}{\partial \theta_{R}^{2}}\right) .
$$

The eigenvalues of $L_{0}$ and $\bar{L}_{0}$ are the conformal weights of the primary fields and are expressed as

$$
\begin{aligned}
& h_{m n}^{l}=-\frac{l(l+1)}{k-2}+\frac{(m+n k)^{2}}{4 k} \\
& \bar{h}_{m n}^{l}=-\frac{l(l+1)}{k-2}+\frac{(m-n k)^{2}}{4 k} .
\end{aligned}
$$

The term in $F_{\mu \nu}$ that we want to add to our gauged action is given by

$$
S_{a d d}=\frac{k}{\pi} \int d^{2} z X\left(r, \theta_{L}, \theta_{R}\right) \bar{\partial} \partial \phi
$$

On the other hand equation (2.13) yields

$$
\left(\partial_{\theta_{L}}+\partial_{\theta_{R}}\right) X=0
$$

Therefore

$$
X\left(r, \theta_{L}, \theta_{R}\right)=X\left(r, \theta_{L}-\theta_{R}\right) .
$$

This additional term may be regarded as a perturbation to the conformal field theory of the coset model. Hence, we require that the operator $\bar{\partial} \partial \phi X$ has conformal dimension $(1,1)$ with respect to the energy momentum tensor of the unperturbed theory in (2.10). Since $\bar{\partial} \partial \phi$ is already of dimenion $(1,1)$ we must have

$$
L_{0} X=\bar{L}_{0} X=0
$$

Therefore $X$ is a conformal operator of the form (3.13) and of dimension $(0,0)$, and since it depends only on the difference $\theta_{L}-\theta_{R}$ we deduce that 
the winding number $n$ must be zero. Hence $X(r, \theta)$ is given by all the vertex operators

$$
X(r, \theta)=P_{\frac{1}{2} m,-\frac{1}{2} m}^{l}(\cosh r) e^{\frac{i}{2} m\left(\theta_{L}-\theta_{R}\right)},
$$

for which the representations and the level $k$ have to satisfy

$$
-\frac{l(l+1)}{k-2}+\frac{m^{2}}{4 k}=0 .
$$

To summarise, we have modified the gauged WZWN model by a term involving the field strengh $F_{\mu \nu}$ of the $U(1)$ gauge group. We found that the addition of this term changes completely the singularity structure of the twodimensional target space. We have calculated explicitly the scalar curvature and found the singularity for a particular case of the additional term. The conformal field theory of the black hole in the persence of the $F_{\mu \nu}$ term was also analysed. In particular, this term can be treated as a perturbation by a $(1,1)$ conformal operator of the $S L(2, R) / U(1)$ coset model.

Acknowledgements : I would like to thank K. S. Narain, E. Gava, H. Sarmadi, S. Panda and B. Rai for many useful discussions. The financial support from IAEA and UNESCO is also hereby acknowledged. 


\section{Bibliography}

[1] E. Brézin, V. Kazakov and Al. B. Zamolodchikov, Nucl. Phys. B338 (1990) 673.

[2] D. J. Gross and N. Miljković, Phys. Lett. B238 (1990) 217.

[3] P. Ginsparg and J. Zinn-Justin, Phys. Lett. B240 (1990) 333.

[4] G. Parisi, Phys. Lett. B238 (1990) 209.

[5] D. J. Gross and I. R. Klebanov, Nucl. Phys. B344 (1990) 475; Nucl. Phys. B354 (1991) 459.

[6] J. Polchinsky, Nucl. Phys. B346 (1990) 253.

[7] S. R. Das and A. Jevicki, Mod. Phys. Lett. A5 (1990) 1639.

[8] A. Sengupta and S. Wadia, 'Excitations and Interactions in $d=1$ String Theory', Tata preprint TIFR/TH/90-13 (1990).

[9] D. J. Gross and I. R. Klebanov, Nucl. Phys. B352 (1991) 671.

[10] M. Goulian and Li, Santa Barbara preprint UCSBTH-90-61.

[11] D. Kutasov and P. Di Francesco, Princeton preprint PUPT-1237.

[12] Y. Kitazama, Harvard preprint HUTP-91/A013.

[13] Vl. S. Dotsenko, Paris preprint PAR-LPTHE-91-18.

[14] G. Mandal, A. Sengupta and S. Wadia, IAS preprint IASSNS$\mathrm{HEP} / 91 / 8$. 
[15] K. Demeterfi, A. Jevicki and J. P. Rodrigues, Brown preprint BROWNHEP-795 (1991).

[16] J. Polchinsky, Texas preprint UTTG-06-91.

[17] G. Moore, 'Double Scaled Field Theory at $c=1$ ', Rutgers preprint RU-91-12.

[18] I. R. Klebanov and D. A. Lowe, 'Correlation Functions in Two Dimensional Quantum Gravity Coupled to a Compact Scalar Field', Princeton preprint PUPT-1256 (1991).

[19] E. Witten, Phys. Rev. D44 (1991) 314.

[20] S. Elitzur, A. Forge and E. Rabinovici, 'Some Global Aspects of String Compactifications', Hebrew University preprint RI-143/90;

G. Mandal, A. M. Sengupta and S. Wadia, 'Classical Solutions in 2Dimensional String Theory'; IAS preprint IASSNS-HEP/91/10;

M. Rocek, K. Schoutens and A. Sevrin, IAS preprint IASSNS-HEP91/14;

I. Bar, USC-91/HEP (1991).

[21] E. Braaten, T. L. Curtright and C. K. Zachos, Nucl. Phys. B260 (1985) 630 .

[22] I. Jack, D. R.T. Jones, N. Mohammedi and H. Osborn, Nucl. Phys. B332 (1990) 359.

[23] C. M. Hull and B. Spence, Phys. Lett. B232 (1989) 204.

[24] L. Alvarez-Gaumé, D. Z. Freedman and S. Mukhi, Ann. Phys. (N.Y.) 134 (1981) 85;

S. Mukhi Nucl. Phys. B264 (1986) 640.

[25] D. Karabali, Q.-H. Park, H. J. Schnitzer and Z. Yang, Phys. Lett. B216 (1989) 307;

H. J. Schnitzer, Nucl. Phys. B324 (1989) 412;

D. Karabali and H. J. Schnitzer, Nucl. Phys. B329 (1990) 649; 
K. Gawedzki and A. Kupianen, Phys. Lett. B215 (1988) 119; Nucl. Phys. B320(FS) (1989) 649.

[26] R. Dijkgraaf, H. Verlinde and E. Verlinde, 'String propagation in a Black Hole Geometry', PUPT-1252, IASSNS-HEP-91/22 (1991). 